\title{
EQUAÇÕES DE VOLUME E DE TAPER PARA QUANTIFICAR MULTIPRODUTOS DA MADEIRA EM FLORESTA ATLÂNTICA ${ }^{1}$
}

\author{
José Franklin Chichorro², José Luiz Pereira Resende ${ }^{3}$ e Helio Garcia Leite ${ }^{4}$
}

\begin{abstract}
RESUMO - Este estudo foi conduzido com o objetivo de ajustar uma equação de volume, a partir do modelo logaritmizado de Schumacher \& Hall (1933), para estimar o volume comercial, e uma equação de afilamento (taper), a partir do modelo de Demaerschalk (1972), para estimar a quantidade de multiprodutos de madeira de espécies da Floresta Atlântica. Os dados foram coletados na Gleba C da Floresta Nacional do Rio Preto - IBAMA, no município de Conceição da Barra-ES. Foram mensuradas 165 árvores em pé, subdivididas em dez classes de diâmetro, com qualidade de fuste QF1 e pertencentes às espécies com maior VI e, ou, espécies de valor comercial. Essas árvores reuniram 62 espécies e 38 famílias. A equação de volume ajustou-se bem aos dados, com $\bar{R}^{2}$ igual a 0,9815 e resíduos normalmente distribuídos. A equação de taper também ajustou-se bem aos dados, com $\bar{R}^{2}$ igual a 0,9346 e distribuição gráfica dos resíduos normal. Pelo teste F (Graybill, 1976), constatou-se que houve igualdade entre os diâmetros observados e os diâmetros estimados pela equação de taper. A partir de transformações algébricas da equação de taper, foram estimados a altura para um determinado diâmetro superior $d$ e o volume do tronco ou de parte dele. Em seguida, podem ser estimados o número de toras e o respectivo volume, em função do comprimento da tora, do diâmetro superior $d$ mínimo e do uso desejado, o que permite avaliar a árvore em pé.
\end{abstract}

Palavras-chave: Floresta Atlântica, equação de volume, equação de taper e volume comercial.

\section{VOLUME AND TAPER EQUATIONS FOR WOOD MULTIPRODUCTS QUANTIFICATION IN FLORESTA ATLANTICA-BRAZIL}

\begin{abstract}
This study was carried out to estimate a volume equation from a logarithmic model of Schumacher \& Hall (1933) for comercial volume determination and a taper equation from the model of Demaerschalk (1972), to estimate wood multiproducts yield in Floresta Atlântica. Data were collected in Gleba C (C Glebe), with 289.5 hectares, owned by Floresta Nacional do Rio Preto (Rio Preto National Forest), under the administration of IBAMA, located in Conceição da Barra - Espirito Santo, Brazil. A total of 165 standing trees were measured, subdivided into 10 diameter classes, with bole quality QF1, with the greatest VI, and/or species of commercial value. These trees represented 62 species and 38 families. The volume equation fitted well within the data, with $\bar{R}^{2}$ of 0.9815 , and normally distributed residues. The taper equation also fitted well within the data, with $\bar{R}^{2}$ of 0.9346 and graphic distribution of the residues was normal. The F test (Graybill, 1976) showed equivalence between the observed and estimated diameters by the taper equation. The taper equation algebric transformations allowed to estimate the height for a given superior diameter $\boldsymbol{d}$, and the trunk volume for a desired use, allowing to evaluate the amount of multiproducts for standing trees.
\end{abstract}

Key words: $\quad$ Atlantic Forest, volume equation, taper equation and commercial volume.

1 Recebido para publicação em 21.2.2002.

Aceito para publicação em 4.11.2003.

2 Prof. Adjunto do Dep. de Engenharia Florestal da Universidade Federal de Mato Grosso - UFMT, 78060-900 Cuiabá-MT, <franklim@cpd.ufmt.br>. ${ }^{3}$ Prof. Titular e Pesquisador do Dep. de Engenharia Florestal da Universidade Federal de Lavras UFLA, 37200-000 Lavras-MG, <jlprezen@ufla.br>. ${ }^{4}$ Prof. do Dep. de Engenharia Florestal da Universidade Federal de Viçosa - UFV, 36570-000 Viçosa-MG, <hgleite@ufv.br>. 


\section{INTRODUÇÃO}

A redução drástica do estoque de madeiras tropicais da Floresta Atlântica ocorreu em razão da expansão da agricultura e da exploração de produtos florestais, particularmente de espécies de valor comercial. Essas atividades descaracterizaram e degradaram a cobertura florestal desse ecossistema, que, atualmente, representa apenas 4\% de sua cobertura original (Brasil, 1991; Embrapa, 1994).

A crescente demanda por produtos florestais, a valorização e o desperdício dessas madeiras foram preponderantes na redução do estoque madeireiro desse ecossistema. Acrescentam-se, ainda, o alto custo de implantação e de manutenção e o longo tempo de espera para a maturidade de florestas plantadas.

Por outro lado, os desperdícios causados pelos atuais processos de transformação têm induzido à pesquisa $\mathrm{e}$ ao desenvolvimento de modelos estatísticos aplicados ao manejo de florestas naturais que auxiliem na definição de uso dessas madeiras e de transformação em produto final, com o intuito de torná-las mais rentáveis.

Dentre essas técnicas de modelagem destacam-se os modelos de taper, considerados flexíveis por possibilitarem estimar o diâmetro a uma altura qualquer do tronco, estimar a altura comercial relativa a um diâmetro mínimo de uso, bem como estimar o volume parcial ou total do fuste, via integração da área das seções do tronco (Husch et al., 1972).

Os modelos de taper foram desenvolvidos para ser aplicados em florestas plantadas puras e florestas naturais mistas, em países de clima temperado, para determinação dos volumes comercial e total, da altura comercial e do diâmetro superior (Kozak et al., 1969; Demaerschalk, 1972; Goulding \& Murray, 1976; Burkhart, 1977; Biging, 1984).

No Brasil, o uso dos modelos de taper, em sua maioria, está relacionado a florestas eqüiâneas puras, com espécies Pinus spp. e Eucalyptus spp. (Campos \& Ribeiro, 1982; Guimarães \& Leite, 1992; Schneider et al., 1996). Estes modelos são pouco usados com espécies nativas, e um exemplo é o estudo com Didymopanax morototonii (morototó), realizado por Garcia et al. (1993). Tal fato pode ser explicado pela grande diversidade de espécies e idades que compõem essas florestas e, conseqüentemente, pelos diferentes perfis de troncos, o que resulta em baixa precisão das equações estimadas.

R. Árvore, Viçosa-MG, v.27, n.6, p.799-809, 2003
Porém, Garcia et al. (1993) consideram os modelos de taper como ferramentas importantes na obtenção de estimativas mais realísticas do volume de partes do tronco, bem como na estimação do diâmetro ao longo do tronco e na estimação da altura até um específico diâmetro superior comercial.

Assim, parecem relevantes estudos de taper que visam definir melhor os multiprodutos de um mesmo tronco para o processamento industrial, aumentando, conseqüentemente, seu aproveitamento.

Portanto, o objetivo geral deste estudo foi ajustar equações de volume e de taper que auxiliassem na definição de multiprodutos para espécies provenientes da Floresta Atlântica. O ajuste, para ambos os modelos, não teve caráter comparativo de precisão entre equações para a escolha da melhor, mas sim o objetivo de obter equações que proporcionassem estimativas de resultados coerentes e aceitáveis estatisticamente, para totalização do volume comercial com casca, e a estimativa de diâmetros em qualquer ponto, ao longo do tronco.

Especificamente, objetivou-se:

- Ajustar um modelo de regressão, visando estimar volume comercial das árvores.

- Ajustar um modelo de taper que estime diâmetros ao longo do tronco das árvores.

- Propor uma alternativa que auxilie na definição de multiprodutos de madeiras.

- Gerar tabelas de multiprodutos da madeira, a partir da árvore em pé.

\section{MATERIAL E MÉTODOS}

\section{1. Área de Coleta dos Dados Amostrais}

O estudo foi realizado na Floresta Nacional de Rio Preto - FNRP, município de Conceição da Barra-ES, com área de 2.830 ha, pertencente ao Instituto Brasileiro do Meio Ambiente e dos Recursos Naturais Renováveis IBAMA. Os dados amostrais foram coletados na Gleba C, com 289,5 ha, área de floresta secundária, no estádio avançado de sucessão (Resolução CONAMA № 10 , de $1^{\circ}$ de outubro de 1993).

A vegetação da FNRP, segundo Amorim (1984), pertence à região da Floresta dos Tabuleiros, caracterizada por floresta densa, com exemplares arbóreos de 
grande porte em altura e diâmetro, destacando-se as espécies Hydrogaster trinervis (bomba-d'água), Pouteria spp. (bapebas), Rinorea bahiensis (tambor), Licania sp. (milho-torrado), Joannesia princeps (boleira) e Virola gardneri (bicuíba).

\subsection{Definição e Medição das Variáveis Dendrométricas e Cubagem Rigorosa}

Foram medidas 165 árvores, todas com fuste reto e não-oco, sem danos aparentes, com aproveitamento total, denominado classe de qualidade de fuste QF1. Considerou-se o fuste das árvores no intervalo do dap ou logo acima das sapopemas, até o ponto que fosse possível aproveitá-lo para processamento em serraria.

As alturas e os respectivos diâmetros ao longo do fuste foram obtidos das árvores em pé, com o relascópio de faixa larga de Bitterlich (Finlayson, 1969), procedimento recomendado por Silva (1982).

No inventário das espécies que se enquadravam na classe de diâmetro e no valor de importância $(V I)$ e, ou, no valor comercial, anotou-se o nome comum e mediramse o diâmetro a 1,30 m do solo (dap) e os demais diâmetros ao longo do fuste e as alturas correspondentes, altura comercial e altura total, na escala apropriada.

As árvores medidas foram distribuídas em classes diamétricas, com diâmetro a partir de $10,0 \mathrm{~cm}$ e com amplitude de $10 \mathrm{~cm}$, pertencendo à última classe aquelas árvores com diâmetro igual ou maior que $100,0 \mathrm{~cm}$. Foram amostradas de 18 a 20 árvores por classe de diâmetro, o que não foi possível para as maiores classes, devido à baixa ocorrência de árvores nessas classes de diâmetro na área (Quadro 1).

O volume das seções e do fuste comercial, por árvore e por classe de dap, para todas as árvores, de todas as espécies foi totalizado, usando-se a fórmula de Smaliam (Scolforo \& Figueiredo Filho, 1993).

\subsection{Ajuste e Análise de Precisão da Equação de Volume}

Para estimar o volume comercial individual com casca, usou-se o modelo desenvolvido por Schumacher \& Hall (1933), representado pela expressão: $\operatorname{Ln}\left(V c c_{i}\right)=$ $\operatorname{Ln} \beta_{0}+\beta_{1} \operatorname{Ln}\left(\right.$ dap $\left._{i}\right)+\beta_{2} \operatorname{Ln}\left(H c_{i}\right)+\varepsilon_{i}$, em que $V c c_{i}=$ volume comercial do fuste da árvore $i$, com casca, $\mathrm{em} \mathrm{m}^{3}(i=1$, $2, \ldots, n) ;$ dap $_{i}=$ diâmetro com casca da árvore $i$, medido a 1,30 $\mathrm{m}$ do solo, em $\mathrm{cm} ; H c_{i}=$ altura comercial da árvore, em m; $L n=$ logaritmo natural (base e); $\beta_{j}=$ coeficientes do modelo $(i=0,1$ e 2$)$; e $\varepsilon_{i}=$ erro aleatório.

Antes do ajuste do modelo aplicou-se a análise de correlação simples da variável volume com dap e $H c$. Em seguida, ajustou-se o modelo proposto pelo método dos mínimos quadrados ordinários (MQO), e para a validação da equação ajustada foram considerados o coeficiente de determinação ajustado $\left(\bar{R}^{2}\right)$ e o teste $t$ para os coeficientes. Foi analisada, também, a distribuição gráfica dos resíduos entre os volumes da cubagem rigorosa e os volumes estimados pela equação (Regazzi \& Leite, 1992; Cecon, 1996).

\subsection{Ajuste e Análise de Precisão da Equação de Taper}

Devido à flexibilidade dos modelos de taper para estimar volume, diâmetro e altura e tomando-se como base as informações empíricas e a revisão de trabalhos científicos (Campos \& Ribeiro, 1982; Lima, 1986; Guimarães \& Leite, 1992; Garcia et al., 1993; Leite et al., 1995; Silva, 1996), foram selecionados os modelos de Kozak (Kozak et al., 1969) e Demaerschalk (Demaerschalk, 1972), representados por

$$
\begin{aligned}
& \left(\frac{d_{i}}{d a p}\right)^{2}=\beta_{0}+\beta_{1}\left(\frac{h_{i}}{H t}\right)+\beta_{2}\left(\frac{h_{i}}{H t}\right)^{2}+\varepsilon_{i} \mathrm{e} \\
& \left(\frac{d_{i}}{d a p}\right)^{2}=10^{2 \beta_{0}} \operatorname{dap}^{2 \beta_{1}-2} L_{i}^{2 \beta_{2}} H t^{2 \beta_{3}}+\varepsilon_{i},
\end{aligned}
$$

que podem ser simplificados para:

$$
\begin{aligned}
& d_{i}=d a p \sqrt{\beta_{0}+\beta_{1}\left(\frac{h_{i}}{H t}\right)+\beta_{2}\left(\frac{h_{i}}{H t}\right)^{2}} \mathrm{e} \\
& d_{i}=10^{\beta_{0}} \operatorname{dap}^{\beta_{1}} L^{\beta_{2}} H t^{\beta_{3}}
\end{aligned}
$$

em que

$d_{i}=$ diâmetro com casca a ser estimado, em $\mathrm{cm}$; dap = diâmetro a 1,30 m do solo, em cm; $H t=$ altura total, em $\mathrm{m} ; h_{i}=$ altura comercial onde se encontra o diâmetro $d_{i}$, em m; $L=H t-h i$, distância da altura total até a altura em que ocorre o diâmetro $d i ; \beta_{i}=$ coeficientes do modelo a serem estimados $(i=0,1,2,3)$; e $\varepsilon_{i}=$ erro aleatório.

R. Árvore, Viçosa-MG, v.27, n.6, p.799-809, 2003 
Quadro 1 - Espécies usadas na cubagem, amostradas na Gleba C da FNRP, ordenadas por nome científico Table 1 - Tree species used in the scaling, sampled in plot C of FNRP

\begin{tabular}{|c|c|c|c|c|c|c|c|c|c|c|c|}
\hline \multirow{2}{*}{ Nome Científico } & \multicolumn{10}{|c|}{ Classe de DAP $(\mathrm{cm})$} & \multirow[b]{2}{*}{ Total } \\
\hline & 1 & 2 & 3 & 4 & 5 & 6 & 7 & 8 & 9 & 10 & \\
\hline Alseis floribunda Schott & 1 & & & & & & & & & & 1 \\
\hline Angostura silvestris (Nees \& Mart.) Albuq. & & & & 1 & & 1 & 1 & & & & 3 \\
\hline Aspidosperma cylindrocarpum Müll. Arg. & & & 1 & 1 & & & 1 & & & & 3 \\
\hline Astronium concinnum Schott & & & 2 & 1 & 1 & & & & 2 & & 6 \\
\hline Astronium graveolens Jacq. & & & 1 & & 1 & 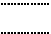 & 2 & 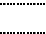 & 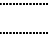 & & 4 \\
\hline Brosimum gaudichaudii Trécul & & & & 1 & & & & & & & 11 \\
\hline Buchenavia rabelloana Mattos & & & & & & & 11 & 1 & & & 2 \\
\hline Byrsonima sp.1 & & & & 1 & & & & & & & 1 \\
\hline Cariniana legalis Kuntze & (a) & & & & 1 & 1 & & & & & 2 \\
\hline Chrysophyllum lucentifolium Cronquist & & 1 & & & & & & & & & 1 \\
\hline Chrysophyllum splendens Spreng. & & 1 & & & & & & & & & 1 \\
\hline Clarisia racemosa Ruiz \& Pav. & ......... & 2 & & 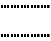 & (1) & & & ................ & 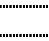 & & 3 \\
\hline Couratari asterotricha Prance & & & 1 & "an' & & & & 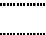 & 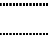 & 1 & 2 \\
\hline Couratari macrosperma A.C. Sm. & & & & & & & & & & 1 & 1 \\
\hline Dialium guianense (Aubl.) Sandwith & 2 & 1 & 1 & 1 & & & & & & & 5 \\
\hline Eriotheca macrophylla (K. Schum.) A. Robyns & & & & 1 & & 2 & & 1 & 1 & 1 & 6 \\
\hline Eschweilera ovata Mart. ex Miers & 2 & 1 & & 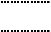 & & & & & & & 3 \\
\hline Eugenia sp. & & 1 & & & & & & & & & 1 \\
\hline Geissospermum laeve Miers & 1 & & & & & & & & & & 1 \\
\hline Goniorrhachis marginata Taub. ex Glaziou & & & & & & 1 & 3 & 3 & 3 & 2 & 12 \\
\hline Hydrogaster trinervis Kuhlm. & & & 1 & 2 & 1 & 2 & 1 & & & 1 & 8 \\
\hline Indeterminada 54 & & & & & 1 & & & & & & 1 \\
\hline Indeterminada 60 & 1 & & & & & & & & & & 1 \\
\hline Inga nuda Salzm. ex Benth. & & 1 & 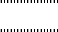 & . & & $\ldots$ & 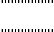 & … & & & 1 \\
\hline Jacaranda sp. & & & & & 1 & & & & & & 1 \\
\hline Joannesia princeps Vell. & & 1 & 2 & & 2 & 2 & 2 & 1 & & & 10 \\
\hline Lacmellea pauciflora (Kuhlm.) Markgr. & 1 & & & & & & & & & & $\begin{array}{ll}1 \\
1\end{array}$ \\
\hline Lecythis lurida (Miers) Mori & & & 1 & & & & & & & & 1 \\
\hline Lecythis pisonis Cambess. & & & & 1 & & & 1 & & & & 2 \\
\hline Licania salzmannii Fritsch & & & & & & & & & & 1 & 1 \\
\hline Lucuma butyrocarpa Kuhlm. & & & & & & 1 & & 2 & 1 & & 4 \\
\hline Luehea mediterranea (Vell.) Angely & & 1 & 11 & & & & 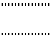 & 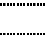 & & & 2 \\
\hline Macrolobium latifolium Vogel & & 1 & & 2 & & & & & & & 3 \\
\hline Manilkara bella Monach. & 1 & 1 & 1 & 2 & 1 & & & & & & 6 \\
\hline Manilkara salzmannii (A. DC.) H.J. Lam. & & & 1 & & & & & & & & 1 \\
\hline Micropholis crassipedicellata Pierre & & & & & & 1 & & & & & 1 \\
\hline Ocotea confertiflora $\mathrm{Mez}$ & & & 1 & & 1 & & & & & & 2 \\
\hline Ocotea odorifera (Vell.) Rohwer & & & & & 1 & & & & & & 1 \\
\hline Oxandra sp. & 2 & & & & & & & & & & 2 \\
\hline Parapiptadenia pterosperma (Benth.) Brenan & & & & & & & 11 & 1 & & & 2 \\
\hline Pouteria aff. hispida Eyma & & & & 1 & & & & & & & 1 \\
\hline Pouteria bullata (S. Moore) Baehni & 1 & & & & & & & & & & 1 \\
\hline Pouteria caelomatica Rizzini & & & & & & 1 & & & & & 1 \\
\hline Protium heptaphyllum March. & 1 & & 1 & & & & & & & & 2 \\
\hline Protium heptaphyllum March. subsp. heptaphyllum & & & 1 & & & & & & & & 1 \\
\hline Protium warmingianum March.. & 1 & 1 & & 1 & 1 & & 1 & & & & 5 \\
\hline Pterocarpus glabratus Kuntze & & & 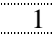 & 1 & & 1 & 1 & 1 & & & 5 \\
\hline Pterygota brasiliensis Allemão & & & & & & & & & 1 & & 1 \\
\hline Schizolobium parahyba (Vell.) Blake & & & & & 1 & & & & & & 1 \\
\hline Schoepfia obliquifolia Turez... & 2 & & 1 & & & & & & & & 3 \\
\hline Simarouba amara Aubl. & & 1 & & & 1 & & & & & & 2 \\
\hline Sloanea sp. & & 1 & & & & & & & & & 1 \\
\hline Spondias macrocarpa Engl. & & & & & 2 & 1 & & & 1 & & 4 \\
\hline Sterculia elata Ducke & & & & & & & & 1 & & & 11 \\
\hline Sterculia speciosa K. Schum. & 2 & 1 & & - & & 2 & 3 & & & & 8 \\
\hline Sweetia fruticosa Spreng. & & & & & 1 & 1 & & & & & 2 \\
\hline Talisia intermedia Radlk. & & & & 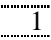 & & & & 1 & & & 2 \\
\hline Tapirira guianensis Aubl. & & 1 & & & & & & & & & 1 \\
\hline Trichilia quadrijuga Kunth & & 1 & & & & & & & & & 1 \\
\hline Vatairea heteroptera (Allemão) Ducke ex de Assis Iglesias & & & & & & & & 1 & & & 1 \\
\hline Vatairiopsis araroba (Aguiar) Ducke & & & & & & 2 & & 1 & 1 & & 4 \\
\hline Virola gardneri Warb. & 1 & 2 & & 1 & 1 & 1 & 1 & & & & 7 \\
\hline Total & 19 & 20 & 18 & 19 & 19 & 20 & 19 & 14 & 10 & 7 & 165 \\
\hline
\end{tabular}


A precisão da equação de taper foi determinada pelo coeficiente de determinação ajustado $\left(\bar{R}^{2}\right)$, pela significância dos coeficientes estimados (teste t) e pelo erro médio em porcentagem (e\%), além da análise gráfica dos resíduos (Regazzi \& Leite, 1992; Cecon, 1996; Silva, 1996).

Considerou-se, também, a estatística $\mathrm{F}$ proposta por Graybill (1976), através do ajuste de regressão linear expressa por: $\operatorname{Dobs}_{i}=\beta_{0}+\beta_{1} \operatorname{Det}_{i}+\varepsilon_{i}$, em que $\operatorname{Dobs}_{i}=$ diâmetros observados ao longo do tronco, a partir de medições, em cm; Dest $_{i}=$ diâmetros estimados ao longo do tronco, pela equação ajustada, $\mathrm{em} \mathrm{cm} ; \beta_{i}=$ coeficientes a serem estimados; e $\varepsilon_{i}=$ erro aleatório.

Esse procedimento consiste em testar a similaridade das variáveis da equação, considerando a seguinte hipótese:

$$
H_{0}: \hat{\beta}=\left[\begin{array}{l}
0 \\
1
\end{array}\right] \text { "versus" } H_{a}: \text { não } H_{0} .
$$

A não-rejeição de $\mathrm{H}_{0}$ implica que os valores observados e estimados são estatisticamente coincidentes, ou seja, $\hat{\beta}_{0}=0$ e $\hat{\beta}_{1}=1$.

O valor da estatística F (Graybill, 1976) foi obtido pela expressão representada por

$$
F\left(H_{0}\right)=\frac{\left(c^{\prime} \hat{\beta}-\theta\right)^{\prime}\left[c^{\prime}\left(X^{\prime} X\right)^{-1} c\right]^{-1}\left(c^{\prime} \hat{\beta}-\theta\right)}{p \cdot \hat{\sigma}^{2}},
$$

em que $X=$ diâmetro estimado; $c^{\prime}=\left[\begin{array}{ll}1 & 0 \\ 0 & 1\end{array}\right]$;

$\beta^{\prime}=\left[\begin{array}{ll}\beta_{0} & \beta_{1}\end{array}\right]=$ vetor transposto dos coeficientes da equação estimada; $\theta^{\prime}=\left[\begin{array}{ll}0 & 1\end{array}\right] ; \mathrm{X}=$ matriz dos diâmetros estimados; $X^{\prime}=$ matriz $X$ transposta; $X^{\prime} X=$ matriz $X$ transposta multiplicada pela matriz $X ; \mathrm{p}=$ posto da matriz $c^{\prime} ; \hat{\sigma}^{2}=$ quadrado médio do resíduo; e $F_{\text {tab }}=[\alpha$; posto de $c^{\prime} ; n$-posto $\left.(X)\right]$, para $(\alpha=0,01)$, (Chichorro, 1999).

Assim, aplicou-se o critério de decisão proposto por Guimarães (1994), conforme as condições e a situação final apresentadas no Quadro 2.

\subsection{Estimativa do Volume de Toras para Diferentes Usos}

O volume das toras por fuste comercial, para conversão em produtos serrados, laminados e energia, visando o maior aproveitamento das árvores, foi estimado com a equação de taper que melhor se ajustou aos dados.
Assim, algumas transformações algébricas na equação de taper para diâmetro foram necessárias, obtendo-se as respectivas equações para altura comercial, onde ocorrem o diâmetro superior $d$ desejado para determinado uso, e a equação para volume total ou de parte do tronco (Lima, 1986). O número de toras foi definido, levando-se em consideração o comprimento e o diâmetro mínimo em função do uso estabelecido.

\section{RESULTADOS}

\section{1. Árvores Selecionadas para Cubagem}

Das 165 árvores medidas, foram contempladas 62 espécies, representando $32,0 \%$ do total de espécies amostradas, e 28 famílias, representando 53,8\% do total, em relação ao inventário do segundo nível de abordagem da Gleba C.

\subsection{Ajuste da Equação de Volume Comercial}

A análise de correlação simples da variável volume com o dap e $H c$ apresentou, respectivamente, os coeficientes de 0,9790 e de 0,7675 , o que indica um alto grau de associação das variáveis explicativas com a variável dependente.

A equação ajustada para estimar o volume comercial individual com casca foi:

$$
\begin{aligned}
\operatorname{Ln}\left(V c c_{i}\right) & =-9,97595150+2,05409213 \cdot \operatorname{Ln}\left(\operatorname{dap}_{i}\right) \\
& +0,87842365 \cdot \operatorname{Ln}\left(H c_{i}\right)
\end{aligned}
$$

Quadro 2 - Critérios utilizados para avaliação do ajuste da equação de taper

Table 2 - Criteria used for evaluating taper equation adjustment

\begin{tabular}{|c|c|c|c|l|}
\hline Caso & Teste $\mathrm{F}$ & \multicolumn{1}{|c|}{$\mathrm{R}^{2}(\%)$} & \multicolumn{1}{|c|}{ Erro (\%) } & \multicolumn{1}{|c|}{ Situação } \\
\hline 1 & n. s. & $\geq 90$ (Alto) & $<10$ (Baixo) & Ideal \\
\hdashline 2 & n. s. & $\geq 90$ (Alto) & $\geq 10$ (Alto) & Aceitável \\
\hdashline 3 & n. s. & $<90$ (Baixo) & $<10$ (Baixo) & Inaceitável \\
\hline 4 & n. s. & $<90$ (Baixo) & $\geq 10$ (Alto) & Inaceitável \\
\hline 5 & sig. & $\geq 90$ (Alto) & $<10$ (Baixo) & Aceitável \\
\hline 6 & sig. & $\geq 90$ (Alto) & $\geq 10$ (Alto) & Inaceitável \\
\hdashline 7 & sig. & $<90$ (Baixo) & $<10$ (Baixo) & Inaceitável \\
\hline 8 & sig. & $<90$ (Baixo) & $\geq 10$ (Alto) & Inaceitável \\
\hline
\end{tabular}

R. Árvore, Viçosa-MG, v.27, n.6, p.799-809, 2003 
A análise de variância da regressão para validação da equação gerou a estatística $F$ significativa $(\mathrm{P}<0,01)$ e $R^{2}$ ajustado igual a 0,9815 . Os coeficientes de regressão foram todos significativos pela estatística $t(\mathrm{P}<0,01)$, com valores de $\mathrm{t}\left(\beta_{0}\right)=-78,4655, t\left(\beta_{1}\right)=58,9433$ e $t\left(\beta_{2}\right)=$ 14,3703 , o que confirma a importância das variáveis explicativas dap e $H c$ na equação.

Os volumes observados versus os volumes estimados e os resíduos desses volumes estão representados graficamente na Figura 1. A Figura 1(a e b) mostra a proximidade entre os volumes estimados e observados, uma vez que eles se distribuem bem próximos à linha de tendência apresentada. A Figura 1(b) comprova graficamente a distribuição normal dos resíduos desses volumes, o que dá suporte às inferências estatísticas do teste de validação.

\subsection{Aplicação da Equação de Volume Comercial}

Com a equação ajustada foram estimados os volumes comerciais com casca para árvores individuais, em função do diâmetro a 1,30 m do solo e da altura comercial (Quadro 3), para todas as espécies. Como na cubagem foram consideradas apenas árvores com qualidade de fuste QF1, as estimativas de volume para essas árvores certamente são mais precisas, o que torna possível estimar com maior segurança o volume de madeira do tronco para uso em serraria e, ou, laminação.

\subsection{Ajuste da Equação de Taper}

Dos dois modelos de taper (Kozak e Demaerschalk) submetidos à análise de regressão (Lima, 1986), optouse pelo modelo de Demaerschalk, por ter sido o mais preciso, de acordo com os resultados das análises estatísticas. Como o ajuste dos modelos de taper visava a obtenção de estimativas precisas e aceitáveis estatisticamente, os resultados foram apresentados apenas para um dos modelos, cuja análise de regressão gerou a seguinte equação:

$$
d=10^{0,09603699} d a p^{0,94493101} L^{0,42390648} H t^{-0,44170002}
$$

O ajuste da equação foi significativo pela estatística $F(P<0,01)$, com $R^{2}$ ajustado igual a 0,9346 . Os coeficientes da regressão foram todos significativos pela estatística $t(P<0,01), \operatorname{com} t\left(\beta_{0}\right)=2,7755, t\left(\beta_{1}\right)=61,5581$, $t\left(\beta_{2}\right)=34,9149$ e $t\left(\beta_{3}\right)=-11,8978$.

Na Figura 2 estão a distribuição dos diâmetros observados versus os diâmetros estimados e a análise da distribuição dos resíduos. A Figura 2(a) mostra que os diâmetros distribuem-se uniformemente ao longo da linha, indicando precisão entre o valor observado e o estimado. A Figura 2(b) mostra a distribuição normal dos resíduos, comprovando as inferências estatísticas tiradas do teste de validação da equação de taper.
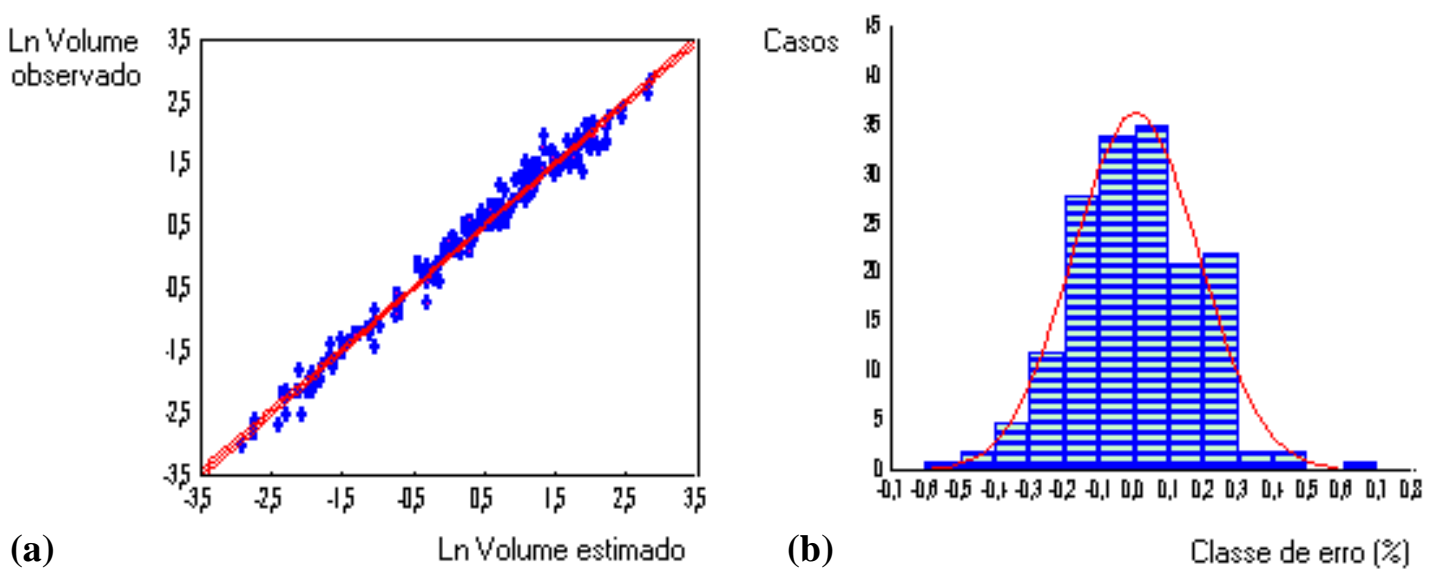

Figura 1 - Volumes observados versus volumes estimados (a) e distribuição dos resíduos puros (b), a partir da equação de volume comercial com casca $(V c)$.

Figure 1 - Observed versus estimated volumes (a) and residual distribution (b), based on commercial volume (with bark) equation (Vc).

R. Árvore, Viçosa-MG, v.27, n.6, p.799-809, 2003 
Quadro 3 - Volumes individuais $\left(\mathrm{m}^{3}\right)$ estimados para árvores de diferentes diâmetros e alturas na Gleba C, da FNRP Table 3 - Estimated tree volume $\left(m^{3}\right)$ for tree species of plot $C$ of FNRP

\begin{tabular}{|c|c|c|c|c|c|c|c|c|c|c|c|c|c|}
\hline \multirow{2}{*}{$\begin{array}{l}\text { DAP } \\
(\mathrm{cm})\end{array}$} & \multicolumn{13}{|c|}{ Altura Comercial (m) } \\
\hline & 6 & 8 & 10 & 12 & 14 & 16 & 18 & 20 & 22 & 24 & 26 & 28 & 30 \\
\hline 15 & 0,0585 & $\mathbf{0 , 0 7 5 3}$ & 0,0916 & 0,1075 & 0,1231 & & & & & & & & \\
\hline 25 & 0,1669 & 0,2149 & 0,2615 & 0,3069 & 0,3514 & 0,3951 & 0,4382 & 0,4807 & & & & & \\
\hline 35 & 0,3332 & 0,4290 & 0,5219 & 0,6125 & 0,7014 & 0,7887 & 0,8746 & 0,9594 & $\mathbf{1 , 0 4 3 2}$ & 1,1261 & & & \\
\hline 45 & 0,5583 & 0,7189 & 0,8745 & 1,0264 & 1,1753 & 1,3215 & 1,4656 & 1,6077 & 1,7481 & 1,8869 & & & \\
\hline 55 & & 1,0856 & 1,3207 & 1,5500 & 1,7748 & 1,9957 & 2,2132 & 2,4278 & 2,6399 & 2,8495 & 3,0571 & & \\
\hline 65 & & 1,5300 & 1,8613 & 2,1846 & 2,5014 & 2,8127 & 3,1193 & 3,4217 & 3,7205 & 4,0161 & 4,3086 & 4,5984 & \\
\hline 75 & & 2,0528 & 2,4973 & 2,9311 & 3,3561 & 3,7738 & 4,1851 & 4,5910 & 4,9919 & 5,3884 & 5,7809 & 6,1697 & \\
\hline 85 & & & 3,2294 & 3,7904 & 4,3400 & 4,8801 & 5,4121 & 5,9369 & 6,4553 & 6,9681 & 7,4757 & 7,9785 & 8,4770 \\
\hline 95 & & & & 4,7633 & 5,4540 & 6,1327 & 6,8012 & 7,4607 & 8,1123 & 8,7566 & 9,3945 & 10,0264 & 10,6528 \\
\hline 105 & & & & 5,8504 & 6,6988 & 7,5324 & 8,3535 & 9,1636 & 9,9638 & 10,7552 & 11,5386 & 12,3148 & 13,0842 \\
\hline 115 & & & & 7,0525 & 8,0751 & 9,0801 & 10,0699 & 11,0463 & 12,0110 & 12,9650 & 13,9094 & 14,8450 & 15,7725 \\
\hline 125 & & & & & & 10,7764 & 11,9511 & 13,1100 & 14,2548 & 15,3871 & 16,5079 & 17,6183 & 18,7191 \\
\hline 135 & & & & & & & 13,9979 & 15,3552 & 16,6962 & 18,0223 & 19,3351 & 20,6357 & 21,9250 \\
\hline
\end{tabular}

Obs.: Os valores em negrito referem-se aos limites da dispersão das árvores-amostra.
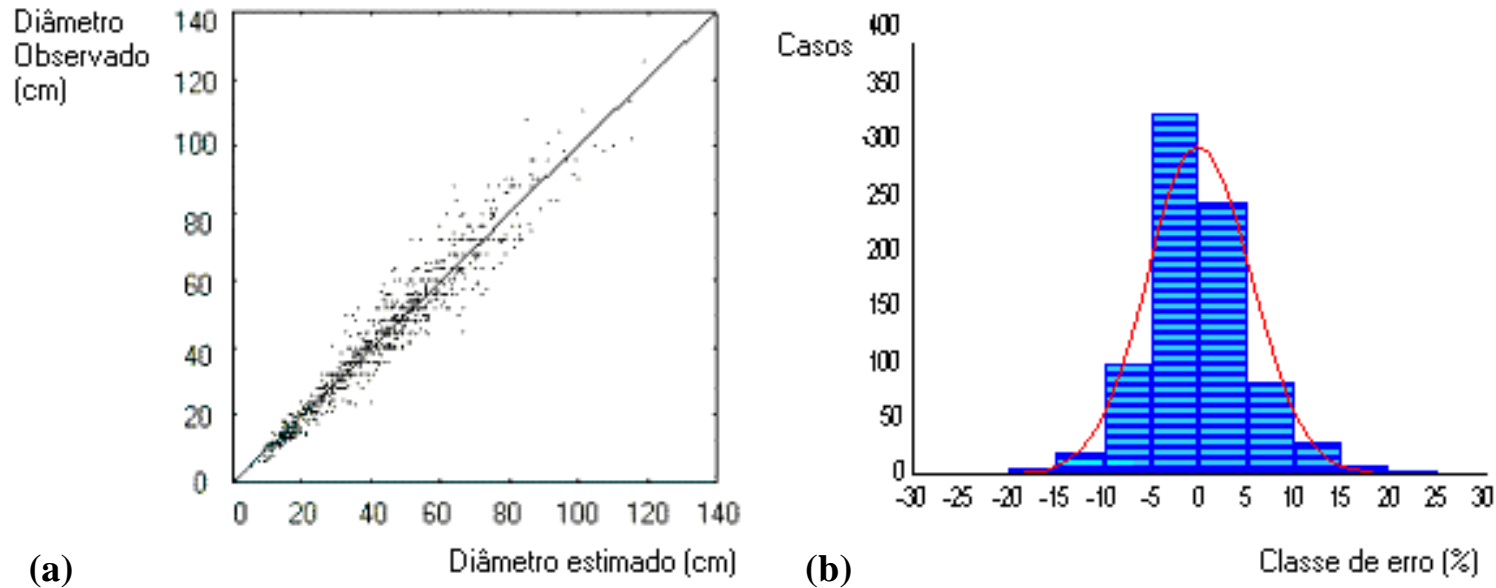

Figura 2 - Diâmetros observados versus estimados (a) e distribuição dos resíduos puros (b), obtidos com a equação de taper. Figure 2-Observed versus estimated diameters (a) and residual distribution (b), obtained from taper equation.

Para aplicar o teste F (Graybill, 1976), ajustou-se o modelo proposto e obteve-se a equação $D o b s_{i}=-$ $0,51772490161+1,0096552003$ Dest $_{i}$, que apresentou a estatística $R^{2}$ ajustado igual a $0,9349, \mathrm{CV}$ igual a 12,58 , e \% igual a $-0,2147$ e $F_{(H O)}$ igual a 0,65 .

O teste $F$ foi não-significativo, portanto não se rejeita $H_{0}$, verificando-se que o coeficiente estimado $\beta_{0}$ é igual a 0 e o $\beta_{1}$ é igual a 1 . Assim, existe similaridade entre os valores de diâmetros observados e dos diâmetros estimados, os quais são estatisticamente coincidentes, perfazendo uma linha reta, que passa pela origem e tem declividade igual a 1.

Os resultados obtidos estão de acordo com o caso 1, definido por Guimarães (1994), com teste $F_{(H O)}$ 
não-significativo, coeficiente de determinação alto $\left(R^{2}\right.$ ajustado $=0,9349)$ e erro médio baixo $(\mathrm{e} \%=-0,1247 \%)$, além do $\mathrm{CV}=12,58 \%$.

Esse teste, segundo Silva (1996), constitui uma forma analítica de realizar a análise de resíduos, uma vez que algumas tendências observadas apenas pela análise visual dos gráficos podem ser enganosas, levando à rejeição de modelos que, na realidade, apresentam estimativas confiáveis.

Quanto à precisão da equação de taper, Demaerschalk (1973), citado por Lima (1986), considera que o mais importante benefício de um sistema compatível é obter resultados consistentes e que a precisão da equação de taper depende da precisão da equação de volume da qual deriva. Considera, também, que a melhor função para estimar taper não é necessariamente a melhor para estimar volume.

Esse modelo, além do bom ajuste, fornece estimativas precisas e resultados consistentes e aceitáveis estatisticamente, na estimação de diâmetros ao longo do tronco.

Bons ajustes de modelos de taper para diâmetro, altura e volume foram obtidos para dados provenientes de florestas eqüiâneas puras ou mistas, onde existe maior regularidade no perfil e nas dimensões dos troncos. Florestas tropicais apresentam grande variedade de espécies, árvores de diferentes idades e formas de troncos, mesmo para árvores com tronco de qualidade QF1.

\subsection{Equação de Taper para Estimar o Volume de Toras para Diferentes Usos}

Para estimar o volume de toras da árvore em pé, considerando os usos em serraria, laminação e energia, foi necessário obter a equação para altura comercial em que ocorre o diâmetro superior $d$, definido em função do uso, e a equação para volume total ou de parte do tronco, apresentadas a seguir.

- Equação para estimar a altura comercial $(h)$

A partir da equação

$$
d=10^{\beta_{0}} D^{\beta_{1}} L^{\beta_{2}} H^{\beta_{3}}
$$

e com algumas manipulações algébricas (Lima, 1986; Chichorro, 1999), obteve-se a equação representada por

$$
\hat{h}=H-\left(d 10^{-\beta_{0}} D^{-\beta_{1}} H^{-\beta_{3}}\right)^{1 / \beta_{2}}
$$

R. Árvore, Viçosa-MG, v.27, n.6, p.799-809, 2003
Substituindo os coeficientes, obteve-se a equação para altura, em que

$$
h=H-\left(d 10^{-0,09603699} D^{-0,94493101} H^{-(-0,44170002)}\right)^{1 / 0,42390648}
$$

- Equação para estimar o volume do tronco ou de parte dele

$$
\text { Partindo da equação }
$$

$\left(\frac{d}{D}\right)^{2}=10^{2 \beta_{0}} D^{2 \beta_{1}-2} L^{2 \beta 2} H^{2 \beta_{3}}$

e após algumas manipulações algébricas, substituição do diâmetro na expressão da área do tronco ao longo do seu eixo para o comprimento $L$ na equação de volume e integrando-se esta expressão (Lima, 1986; Chichorro, 1999), obteve-se a equação para estimar volume, definida por

$$
\hat{V}=K 10^{2 \beta_{0}} D^{2 \beta_{1}} H^{2 \beta_{3}}\left(\frac{\left(H-h_{1}\right)^{2 \beta_{2}+1}-\left(H-h_{2}\right)^{\beta_{2}+1}}{2 \beta_{2}+1}\right)
$$

Com a substituição dos coeficientes, obteve-se a equação para estimar o volume, representada por

$$
\begin{aligned}
V & =K 10^{2(-0,096037)} D^{2(-0,944931)} H^{2(-0,441700)} \\
& \cdot\left(\frac{\left(H-h_{1}\right)^{2(0,423906+1)}-\left(H-h_{2}\right)^{(0,423906+1)}}{2(0,423906)+1}\right)
\end{aligned}
$$

Com a equação (3) estimou-se à que altura do fuste ocorria o diâmetro superior $d$, mínimo, necessário para determinado uso, e, conseqüentemente, o número de toras. Então, com a equação (6) foi estimado o volume referente a essas toras.

Para fazer as estimativas, definiu-se que os fustes, ou parte deles, com qualidade QF1 e com as dimensões mínimas especificadas (diâmetro $d$ e comprimento da tora) seriam destinados ao uso em serraria e laminação, enquanto os fustes de qualidades inferiores (QF2 e QF3) seriam aproveitados na fabricação de dormentes e mourões, ou usados como lenha ou carvão. No entanto, ressalta-se que fustes de qualidade QF2 podem ser aproveitados parcialmente, desde que a espécie tenha valor no mercado. 
O volume estimado e o respectivo número de toras com uso na produção de madeira serrada encontram-se no Quadro 4. Considerou-se a tora com diâmetro mínimo superior $d$ desejado igual a $25,0 \mathrm{~cm}$ e o comprimento igual a 3,0 m. Assim, uma árvore na classe de diâmetro de $85,0 \mathrm{~cm}$ e com a altura de $16,0 \mathrm{~m}$ teve o volume estimado de $3,5978 \mathrm{~m}^{3}$, o que corresponde a quatro toras de 3,0 $\mathrm{m}$ de comprimento.

O mesmo procedimento, com os resultados mostrados no Quadro 5, foi utilizado para estimar o volume e o respectivo número de toras com diâmetro mínimo $d$ desejado, agora com o uso para laminação.

Em florestas secundárias, embora em estádio sucessional avançado, é normal que haja poucas árvores nas maiores classes diamétricas. Essa distribuição é característica do regime de manejo e do sistema de exploração aplicados. Neste caso, como as dimensões das toras (diâmetro e comprimento) para serraria e laminação são próximas, houve semelhança dos resultados estimados nos Quadros 4 e 5.

Quadro 4 - Estimativas do volume $\left(\mathrm{m}^{3}\right)$ e respectivo número de toras de $3,0 \mathrm{~m}$ de comprimento e $d$ comercial igual a $25,0 \mathrm{~cm}$, para uso em serraria, amostradas na Gleba C da FNRP $\underline{1} /$

Table 4 - Volume estimates $\left(\mathrm{m}^{3}\right)$ and number of $3.0 \mathrm{~m}$ long logs and " $d$ " commercial " $d$ " equal to $25,0 \mathrm{~cm}$, for sawn wood production sampled in plot "C" of FNRP

\begin{tabular}{|c|c|c|c|c|c|c|c|c|c|c|c|c|c|c|c|c|c|c|c|c|c|c|}
\hline \multirow{2}{*}{$\begin{array}{c}\text { Altura } \\
\text { Comercial } \\
(\mathrm{m})\end{array}$} & \multicolumn{22}{|c|}{ Classe de Diâmetro $(\mathrm{cm})$ e Número de Toras ( $\mathrm{T}$ ) } \\
\hline & 35 & $\mathrm{~T}$ & 45 & $\mathrm{~T}$ & 55 & $\mathrm{~T}$ & 65 & $\mathrm{~T}$ & 75 & $\mathrm{~T}$ & 85 & $\mathrm{~T}$ & 95 & $\mathrm{~T}$ & 105 & $\mathrm{~T}$ & 115 & $\mathrm{~T}$ & 125 & $\mathrm{~T}$ & 135 & $\mathrm{~T}$ \\
\hline 4 & & & & & & & 0,4153 & 1 & 0,5442 & 1 & & & & & & & & & & & & \\
\hline 6 & & & 0,2390 & 1 & 0,3493 & 1 & 0,4789 & 1 & 0,6277 & 1 & & & & & & & & & & & & \\
\hline 8 & 0,1576 & 1 & 0,2534 & 1 & 0,7372 & 2 & 1,0108 & 2 & 1,3247 & 2 & 1,6782 & 2 & 2,0708 & 2 & 2,5020 & 2 & & & & & & \\
\hline 10 & 0,1624 & 1 & 0,5525 & 2 & 0,8073 & 2 & 1,1070 & 2 & 1,4508 & 2 & 1,8380 & 2 & 2,2679 & 2 & 3,5395 & 3 & 4,2035 & 3 & & & & \\
\hline 12 & 0,1654 & 1 & 0,5827 & 2 & 1,1616 & 3 & 1,5929 & 3 & 2,0875 & 3 & 2,6446 & 3 & 3,2632 & 3 & 3,9427 & 3 & 4,6823 & 3 & 5,4814 & 3 & & \\
\hline 14 & 0,3751 & 2 & 0,8498 & 3 & 1,2418 & 3 & 1,7027 & 3 & 2,6338 & 4 & 3,3366 & 4 & 4,1171 & 4 & 4,9744 & 4 & 5,9075 & 4 & 6,9158 & 4 & 7,9985 & 4 \\
\hline 16 & 0,3842 & 2 & 0,8892 & 3 & 1,5803 & 4 & 2,1670 & 4 & 2,8399 & 4 & 3,5978 & 4 & 4,4394 & 4 & 5,3637 & 4 & 6,3699 & 4 & 8,1908 & 5 & 9,4731 & 5 \\
\hline 18 & 0,3910 & 2 & 1,1400 & 4 & 1,6658 & 4 & 2,5815 & 5 & 3,3832 & 5 & 4,2860 & 5 & 5,2886 & 5 & 6,3898 & 5 & 7,5885 & 5 & 8,8836 & 5 & 10,2744 & 5 \\
\hline 20 & 0,5855 & 3 & 1,1852 & 4 & 1,9949 & 5 & 2,7354 & 5 & 3,5849 & 5 & 4,9134 & 6 & 6,0628 & 6 & 7,3252 & 6 & 8,6993 & 6 & 10,1840 & 6 & 11,7784 & 6 \\
\hline 22 & 0,5968 & 3 & 1,4260 & 5 & 2,0836 & 5 & 3,1480 & 6 & 4,1255 & 6 & 5,2265 & 6 & 6,4491 & 6 & 7,7919 & 6 & 9,2535 & 6 & 10,8328 & 6 & 12,5288 & 6 \\
\hline 24 & 0,6058 & 3 & 1,4751 & 5 & 2,4063 & 6 & 3,2995 & 6 & 4,6264 & 7 & 5,8610 & 7 & 7,2321 & 7 & 8,7379 & 7 & 10,3770 & 7 & 12,1481 & 7 & 14,0499 & 7 \\
\hline 26 & 0,7924 & 4 & 1,7090 & 6 & 2,4971 & 6 & 3,7102 & 7 & 4,8624 & 7 & 6,1600 & 7 & 7,9649 & 8 & 9,6233 & 8 & 11,4285 & 8 & 13,3791 & 8 & 15,4737 & 8 \\
\hline 28 & & & & & & & 4,0951 & 8 & 5,3668 & 8 & 6,7990 & 8 & 8,3895 & 8 & 10,1363 & 8 & 12,0377 & 8 & 14,0923 & 8 & 16,2985 & 8 \\
\hline 30 & & & & & & & & & & & & & & & & & 13,1059 & 9 & 15,3428 & 9 & 17,7448 & 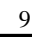 \\
\hline
\end{tabular}

${ }^{1 /}$ Os valores em negrito referem-se aos limites da dispersão das árvores amostradas para cubagem. T = número de toras por árvore.

Quadro 5 - Estimativas do volume $\left(\mathrm{m}^{3}\right)$ e respectivo número de toras de $2,5 \mathrm{~m}$ de comprimento e $d$ comercial igual a $30,0 \mathrm{~cm}$, para uso em laminação, amostradas na Gleba C da FNRP1/

Table 5 - Volume estimates ( $\left.m^{3}\right)$ and number of $2.5 \mathrm{~m}$ long logs and commercial " $d$ " equal to $30.0 \mathrm{~cm}$, for production of veneer sampled in plot "C" of FNRP

\begin{tabular}{|c|c|c|c|c|c|c|c|c|c|c|c|c|c|c|c|c|c|c|c|c|c|c|}
\hline \multirow{2}{*}{$\begin{array}{c}\text { Altura } \\
\text { Comercial } \\
(\mathrm{m})\end{array}$} & \multicolumn{22}{|c|}{ Classe de Diâmetro $(\mathrm{cm})$ e Número de Toras ( $\mathrm{T}$ ) } \\
\hline & 35 & $\mathrm{~T}$ & 45 & $\mathrm{~T}$ & 55 & $\mathrm{~T}$ & 65 & $\mathrm{~T}$ & 75 & $\mathrm{~T}$ & 85 & $\mathrm{~T}$ & 95 & $\mathrm{~T}$ & 105 & $\mathrm{~T}$ & 115 & $\mathrm{~T}$ & 125 & $\mathrm{~T}$ & 135 & $\mathrm{~T}$ \\
\hline 4 & & & & & & & 0,3383 & 1 & 0,4434 & 1 & & & & & & & & & & & & \\
\hline 6 & & & 0,1878 & 1 & 0,2744 & 1 & 0,3762 & 1 & 0,4931 & 1 & & & & & & & & & & & & \\
\hline 8 & & & 0,1962 & 1 & 0,6411 & 2 & 0,8791 & 2 & 1,1521 & 2 & 1,4595 & 2 & 1,8009 & 2 & 2,1759 & 2 & & & & & & \\
\hline 10 & & & 0,4692 & 2 & 0,6856 & 2 & 1,3031 & 3 & 1,7077 & 3 & 2,1634 & 3 & 2,6695 & 3 & 3,2254 & 3 & 3,8304 & 3 & & & & \\
\hline 12 & 0,1266 & 1 & 0,4883 & 2 & 1,0263 & 3 & 1,4073 & 3 & 1,8443 & 3 & 2,7975 & 4 & 3,4519 & 4 & 4,1706 & 4 & 4,9530 & 4 & 5,7984 & 4 & & \\
\hline 14 & 0,1277 & 1 & $\mathbf{0 , 7 3 7 7}$ & 3 & 1,0779 & 3 & 1,8267 & 4 & 2,3940 & 4 & 3,0329 & 4 & 3,7423 & 4 & 5,0523 & 5 & 6,0001 & 5 & 7,0242 & 5 & 8,1238 & 5 \\
\hline 16 & 0,1284 & 1 & 0,7631 & 3 & 1,4062 & 4 & 2,2150 & 5 & 2,9028 & 5 & 3,6775 & 5 & 4,5377 & 5 & 5,4825 & 5 & 6,5110 & 5 & 7,6223 & 5 & 8,8156 & 5 \\
\hline 18 & 0,1289 & 1 & 0,7821 & 3 & 1,7095 & 5 & 2,3442 & 5 & 3,3832 & 6 & 4,2860 & 6 & 5,2886 & 6 & 6,3898 & 6 & 7,5885 & 6 & 8,8836 & 6 & 10,2744 & 6 \\
\hline 20 & 0,1292 & 1 & 1,0296 & 4 & 1,7823 & 5 & 2,7354 & 6 & 3,5849 & 6 & 4,5416 & 6 & 6,0070 & 7 & 7,2577 & 7 & 8,6192 & 7 & 10,0903 & 7 & 11,6699 & 7 \\
\hline 22 & 0,3274 & 2 & 1,0529 & 4 & 2,0836 & 6 & 2,8571 & 6 & 4,0733 & 7 & 5,1603 & 7 & 6,3675 & 7 & 8,0956 & 8 & 9,6143 & 8 & 11,2552 & 8 & 13,0172 & 0 \\
\hline 24 & 0,3293 & 2 & 1,2913 & 5 & 2,1554 & 6 & 3,2499 & 7 & 4,5429 & 8 & 5,7552 & 8 & 7,1015 & 8 & 8,5801 & 8 & 10,1897 & 8 & 11,9288 & 8 & 14,3265 & 0 \\
\hline 26 & 0,3309 & 2 & 1,3177 & 5 & 2,4551 & 7 & 3,6265 & 8 & 4,7527 & 8 & 6,3310 & 9 & 7,8120 & 9 & 9,4386 & 9 & 11,2092 & 9 & 13,1223 & 9 & 15,1766 & 9 \\
\hline 28 & & & & & & & 3,7599 & 8 & 5,2292 & 9 & 6,6246 & 9 & 8,5034 & 10 & 10,2739 & 10 & 12,2012 & 10 & 14,2836 & 10 & 16,5198 & 10 \\
\hline 30 & & & & & & & & & & & & & & & & & 13,1704 & 11 & 15,4183 & 11 & 17,8321 & 11 \\
\hline
\end{tabular}

1/ Os valores em negrito referem-se aos limites da dispersão das árvores amostradas para cubagem. T = número de toras por árvore. 
Quadro 6 - Estimativas do volume $\left(\mathrm{m}^{3}\right)$ e respectivo número de toras de $2,0 \mathrm{~m}$ de comprimento e $d$ comercial igual a $5,0 \mathrm{~cm}$, para uso em energia, amostradas na Gleba $\mathrm{C}$ da $\mathrm{FNRP}^{1 /}$

Table 6 - Volume estimates (m3) and number of $2.0 \mathrm{~m}$ long logs and commercial "d" equal to 5.0cm, for energy use in plot $C$ of FNRP

\begin{tabular}{|c|c|c|c|c|c|c|c|c|c|c|c|c|c|c|c|c|c|c|c|c|c|c|c|c|c|c|}
\hline \multirow{2}{*}{$\begin{array}{c}\text { Altura } \\
\text { Comercial } \\
\text { (m) }\end{array}$} & \multicolumn{26}{|c|}{ Classe de Diâmetro $(\mathrm{cm})$ e Número de Toras ( T ) } \\
\hline & 15 & $\mathrm{~T}$ & 25 & $\mathrm{~T}$ & 35 & $\mathrm{~T}$ & 45 & $\mathrm{~T}$ & 55 & $\mathrm{~T}$ & 65 & $\mathrm{~T}$ & 75 & $\mathrm{~T}$ & 85 & $\mathrm{~T}$ & 95 & $\mathrm{~T}$ & 105 & $\mathrm{~T}$ & 115 & $\mathrm{~T}$ & 125 & $\mathrm{~T}$ & 135 & $\mathrm{~T}$ \\
\hline 4 & 0,0152 & 1 & 0,0399 & 1 & 0,0754 & 1 & 0,1213 & 1 & 0,1773 & 1 & 0,2431 & 1 & & & & & & & & & & & & & & \\
\hline 6 & 0,0406 & 2 & 0,1066 & 2 & 0,2014 & 2 & 0,3238 & 2 & 0,4732 & 2 & 0,6488 & 2 & 0,8503 & 2 & & & & & & & & & & & & \\
\hline 8 & $\mathbf{0 , 0 6 3 3}$ & 3 & 0,1661 & 3 & 0,3138 & 3 & 0,5045 & 3 & 0,7372 & 3 & 1,0108 & 3 & 1,3247 & 3 & 1,6782 & 3 & 2,0708 & 3 & 2,5020 & 3 & & & & & & \\
\hline 10 & 0,0847 & 4 & 0,2224 & 4 & 0,4201 & 4 & 0,6754 & 4 & 0,9869 & 4 & 1,3533 & 4 & 1,7736 & 4 & 2,2469 & 4 & 2,7725 & 4 & 3,3498 & 4 & 3,9782 & 4 & & & & \\
\hline 12 & 0,1055 & 5 & 0,2769 & 5 & $\mathbf{0 , 5 2 3 0}$ & 5 & 0,8410 & 5 & 1,2288 & 5 & 1,6850 & 5 & 2,2082 & 5 & 2,7975 & 5 & 3,4519 & 5 & 4,1706 & 5 & 4,9530 & 5 & 5,7984 & 5 & & \\
\hline 14 & 0,1258 & 6 & 0,3303 & 6 & 0,6238 & 6 & 1,0030 & 6 & 1,4656 & 6 & 2,0097 & 6 & 2,6338 & 6 & 3,3366 & 6 & 4,1171 & 6 & 4,9744 & 6 & 5,9075 & 6 & 6,9158 & 6 & 7,9985 & 6 \\
\hline 16 & 0,1458 & 7 & 0,3829 & 7 & 0,7231 & 7 & 1,1627 & 7 & 1,6989 & 7 & 2,3296 & 7 & 3,0530 & 7 & 3,8677 & 7 & 4,7725 & 7 & 5,7662 & 7 & 6,8479 & 7 & 8,0166 & 7 & 9,2717 & 7 \\
\hline 18 & 0,1656 & 8 & 0,4348 & 8 & 0,8213 & 8 & 1,3206 & 8 & 1,9296 & 8 & 2,6459 & 8 & 3,4675 & 8 & 4,3929 & 8 & 5,4205 & 8 & 6,5491 & 8 & $\mathbf{7 , 7 7 7 7}$ & 8 & 9,1051 & 8 & 10,5306 & 8 \\
\hline 20 & & & 0,4864 & 9 & 0,9186 & 9 & 1,4770 & 9 & 2,1582 & 9 & 2,9594 & 9 & 3,8784 & 9 & 4,9134 & 9 & 6,0628 & 9 & 7,3252 & 9 & 8,6993 & 9 & 10,1840 & 9 & 11,7784 & 9 \\
\hline 22 & & & 0,5375 & 10 & 1,0152 & 10 & 1,6324 & 10 & 2,3852 & 10 & 3,2707 & 10 & 4,2864 & 10 & 5,4302 & 10 & 6,7005 & 10 & 8,0956 & 10 & 9,6143 & 10 & 11,2552 & 10 & 13,0172 & 10 \\
\hline 24 & & & & & 1,1112 & 11 & 1,7868 & 11 & 2,6108 & 11 & 3,5801 & 11 & 4,6918 & 11 & 5,9439 & 11 & 7,3343 & 11 & 8,8615 & 11 & 10,5238 & 11 & 12,3199 & 11 & 14,2486 & 11 \\
\hline 26 & & & & & 1,2068 & 12 & 1,9404 & 12 & 2,8353 & 12 & 3,8879 & 12 & 5,0952 & 12 & 6,4549 & 12 & 7,9649 & 12 & 9,6233 & 12 & 11,4285 & 12 & 13,3791 & 12 & 15,4737 & 12 \\
\hline 28 & & & & & & & & & & & 4,1943 & 13 & 5,4968 & 13 & 6,9637 & 13 & 8,5927 & 13 & 10,3818 & 13 & 12,3293 & 13 & 14,4336 & 13 & 16,6932 & 13 \\
\hline 30 & & & & & & & & & & & & & & & & & & & & & 13,2264 & 14 & 15,4838 & 14 & 17,9079 & 14 \\
\hline
\end{tabular}

${ }^{1 /}$ Os valores em negrito referem-se aos limites da dispersão das árvores amostradas para cubagem. $\mathrm{T}$ = número de toras por árvore.

As árvores das outras qualidades de fuste e sem valor comercial para serraria e laminação devem ser usadas como energia, e, da mesma forma, foram estimados o número de toras e o respectivo volume, mostrados no Quadro 6.

A parte do tronco a partir do diâmetro superior $d$ menor que o mínimo estabelecido para serraria e, ou, laminação pode ser convertida em energia ou destinada a outro uso.

Considerando que a equação de taper permite estimar o volume de madeira do tronco da árvore em pé, para o uso mais adequado, o potencial volumétrico também pode ser estimado economicamente, desde que seja conhecido o valor do metro cúbico de madeira.

\section{CONCLUSÕES}

A análise realizada permite concluir que:

- A equação de volume ajustou-se bem aos dados observados, conforme as estatísticas consideradas para validação, e apresentou estimativas precisas.

- A equação de volume permite gerar uma tabela de dupla entrada, para estimar a quantidade de madeira comercial em $\mathrm{m}^{3} / \mathrm{ha}$.

- O modelo de taper de Demaerschalk gerou equação para estimar diâmetros ao longo do tronco, com precisão conforme estatísticas consideradas para validação.
- A equação de taper ajustada permitiu estimar o diâmetro superior $d$, útil na definição de uso da tora e na conversão em multiprodutos.

- Com algumas transformações algébricas da equação de taper ajustada, foi possível estimar o número de toras e o volume correspondente, avaliando-se a árvore em pé, considerando o uso para serraria, laminação e energia.

- Estudos desta natureza permitem ao manejador da floresta estimar o volume comercial de madeira e também o volume aproveitável do tronco conforme o uso desejado, em função do comprimento da tora e de seu diâmetro superior $d$ mínimo.

\section{REFERÊNCIAS BIBLIOGRÁFICAS}

AMORIM, H. G. Inventário das florestas nativas dos Estados do Rio de Janeiro e Espírito Santo. Brasília: MA/ IBDF/DE, 1984. 204 p.

BIGING, G. S. Taper equations for second mixed-conifers of Northean California. Forest Science, v. 30, n. 4, p. 1103 1117, 1984.

BRASIL. Presidência da República. Brasília: Secretaria da Imprensa. O desafio do desenvolvimento sustentável. Relatório do Brasil para a conferência das Nações Unidas sobre meio ambiente e desenvolvimento. Brasília CIMA Comissão Interministerial para preparação da Conferência das Nações Unidas sobre o meio ambiente e desenvolvimento, 1991. $204 \mathrm{p}$. 
BURKHART, H. E. Cubic-foot volume of Loblolly Pine to any merchantable top limit. Southern Journal of Applied Forestry, v. 1, n. 2, p. 7-9, 1977.

CECON, P. R. Métodos estatísticos I. Viçosa: UFV, 1996. não publicado

CHICHORRO, J. F. Análise estrutural e econômica de multiprodutos da madeira em florestas naturais. 1999. $241 \mathrm{f}$. Tese (Doutorado em Ciência Florestal) Universidade Federal de Viçosa, Viçosa, 1999.

CAMPOS, J. C. C.; RIBEIRO, J. C. Avaliação de dois modelos de taper em árvores de Pinus patula. Revista Árvore, v. 6, n. 2, p. 140-149, 1982.

DEMAERSCHALK, J. P. Converting volume equations to compatible taper equations. Forest Science, v. 18, n. 3, p. 241-245, 1972.

EMBRAPA Atlas do meio ambiente do Brasil. Brasília: Terra Viva, 1994. 130 p.

FINLAYSON, W. The relascope: Bitterlich's spiegel relaskop. Edinburgh: s.n., 1969. 32 p.

GARAY, L. Tropical forest utilization system. VIII. A taper model for entire stemprofile including buttressing. Coll. Forest. Resour., Inst. Forest Prod. Univ. Wash., 1979. 64 p. (contrib., 36).

GARCIA, S. L. R.; LEITE, H. G.; YARED, J. A. G. Análise do perfil do tronco de morototó (Didymoopanax morototonii) em função do espaçamento. In: CONGRESSO FLORESTAL PANAMERICANO e CONGRESSO FLORESTAL BRASILEIRO, 1993, Curitiba. Anais... Curitiba: Sociedade Brasileira de Silvicultura e Sociedade Brasileira de Engenheiro Florestais. 1993. v. 2, p. 485-491.

GOUlding, C. J.; MURRAY, J. C. Polinomial taper equations that are compatible with tree volume equations. New Zealand Jounal of Forestry Science, v. 5, n. 3, p. 313-322, 1976.

GRAYBILL, F. A. Theory and application of linear model. s.1.: Belmont Duxbury, 1976. 704 p.

GUIMARÃES, D. P. Desenvolvimento de um modelo de distribuição diamétrica de passo invariante para prognose e projeção da estrutura de povoamentos de eucalipto. 1994. $160 \mathrm{f}$. Tese (Doutorado em Ciência Florestal) - Universidade Federal de Viçosa, Viçosa, 1994.
GUIMARÃES, D. P.; LEITE, H. G. Um novo modelo para descrever o perfil do tronco. Revista Árvore, v. 16, n. 2, p. 170-180, 1992.

HUSCH, B.; MILLER, C. I.; BEERS, T. W. Forest mensuration. 2.ed. New York: The Ronald Press Company, 1972. 410 p.

KOZAK, A.; MUNRO, D. D.; SMITH, J. G. H. Taper function and their application in forest inventory. The Forestry Chronicle, v. 45, n. 4, p. 278-283, 1969.

LEITE, H. G.; GUIMARÃES, D. P.; CAMPOS, J. C. C. Descrição e emprego de um modelo para estimar múltiplos volumes de árvores. Revista Árvore, v. 19, n. 1, p. 65-79, 1995.

LIMA, F. S. Análise de funções de "taper" destinadas à avaliação de multiprodutos de árvores de Pinus elliottii. 1986. 79 f. Dissertação (Mestrado em Ciência Florestal) Universidade Federal de Viçosa, Viçosa, 1986.

LOETSCH, F.; ZÖHRER, F.; HALLER, K. E. Forest inventory. München: BLV, 1973. v.2. 469 p.

REGAZZI, A. J.; LEITE, H. G. Análise de regressão: teoria e aplicações em manejo florestal. Viçosa, UFV/SIF, 1992. 236 p. (Apostila).

SCHNEIDER, P. R. et al. Forma de tronco e sortimentos de madeira de Eucalyptus grandis Maiden para o estado do Rio Grande do Sul. Ciência Florestal, v. 6, n. 1, p. 79-88, 1996.

SCHUMACHER, F. X.; HALL, F. S. Logarithmic expression of timber-tree volume. Journal of Agricultural Research, v. 47, n. 9, p. 719-734, 1933.

SCOLFORO, J. R. S.; FIGUEIREDO FILHO, A. Volumetria. Lavras, ESAL/ FAEPE, 1993. 126 p.

SILVA, G. F. Alternativas para estimar o volume comercial em árvores de eucalipto. $1996.87 \mathrm{f}$. Dissertação (Mestrado em Ciência Florestal) - Universidade Federal de Viçosa, Viçosa, 1996.

SILVA, J. A. Aplicação do relascópio de banda larga em inventários de florestas tropicais. In: CONGRESSO NACIONAL SOBRE ESSÊNCIAS NATIVAS, 1982, Campos do Jordão. Anais... Campos do Jordão: Silvicultura em São Paulo, v. 16A, n. 1, p. 601-603, 1982.

VANCLAY, G. K. Modelling forest growth and yield: applications to mixed tropical forests. Wallingford: CAB INTERNATIONAL, 1994. $312 \mathrm{p}$.

R. Árvore, Viçosa-MG, v.27, n.6, p.799-809, 2003 